\title{
Scattering theory for dipole quantum fields
}

\author{
Hanno Gottschalk
}

August 13, 2018

\author{
Institut für angewandte Mathematik, Wegelerstr. 6, D-53115 Bonn, Germany \\ gottscha@wiener.iam.uni-bonn.de
}

\begin{abstract}
In the present work a general frame for the scattering theory of local, relativistic dipole quantum fields is presented and some models of interacting dipole fields are considered, i.e. local, relativistic quantum fields with indefinite metric which asymptotically do not converge to free fields, but to free dipole fields. Also, we give explicit formulae for the (nontrivial) scattering matrix of dipole in- and outfields for these models. Furthermore we show how related dipole degrees of freedom occur in the perturbation theory of certain two dimensional models, e.g. massive sine-Gordon or sinh-Gordon models.
\end{abstract}

Keywords: Dipole fields, QFT with indefinite metric, asymptotic states. MSC (2000) 81T05, $81 \mathrm{~T} 08$

\section{Introduction}

In positive metric quantum field theory (QFT) the existence of asymptotic states follows from the Wightman axioms and assumptions on the mass spectrum of the two-point function (first and second mass-gap), cf. [10, 18, 19]. The proof, however, relies crucially on the positivity of Wightman functions.

The Wightman framework of local, relativistic quantum field theory (QFT) turned out to be too narrow for theoretical physicists, who were interested in handling situations involving in particular gauge fields (like in quantum electrodynamics). For several reasons which are intimately connected with the needs of the standard procedure of the perturbative calculation of the scattering matrix (for a detailed discussion, see [22]), the concept of QFT with indefinite metric was introduced [17].

The possible asymptotic behaviour of interacting quantum fields with indefinite metric is not restricted to the usual free fields: Other possible candidates are free dipole fields (and their generalizations) which are local, relativistic quantum fields with a genuinely indefinite metric on the space of states generated from the vacuum. Such fields have been studied in a number of articles, cf. [16] and references therein. 
The main motivation for the study of dipole quantum fields is threefold: (i) massless dipole fields in $d=4$ dimensions have logarithmic increase at spatially separated arguments that is expected for fields that expose confinement [16]; (ii) dipole degrees of freedom occur gauges beyond the Feynman gauge in the Gupta-Bleuler Formalism [21]; (iii) in the perturbation theory of certain models with exponential [12] or trigonometric [6] interaction dipole degrees of freedom occur in perturbation theory - although not present in the completely summed theory. To make sense of the results of perturbation theory one requires a formalism that is capable to describe also the scattering of dipole fields. This will be explained in section 6 of this work.

In [5, 8] local, relativistic and interacting quantum fields have been constructed with indefinite metric state space. These models generalize the class of models studied in [2, 3, 4, 7]. In [5] conditions have been given, when such QFT models have a welldefined (and nontrivial) scattering behaviour with the asymptotic fields given by usual free fields. For these cases a general framework of scattering theory has been proposed in [1. On the other hand, there are fields in the class studied in [5, 8] which have HaagRuelle like scattering amplitudes diverging polynomially in time. The same occurs in the perturbation theory of exponential models in $d=2$.

In this article it is demonstrated that in the simplest case this ill-defined scattering behaviour can be understood in the sense that dipole degrees of freedom have been neglected: Taking free dipole fields instead of usual free fields as asymptotics of the interacting quantum field models leads to a well-defined scattering behaviour.

The article is organized as follows: In Section 2 dipole quantum fields are introduced. Then the scattering theory withe dipole field as asymptotic fields is developed (Section 3). In Section 4 asymptotic states for dipole fields are being constructed via construction of the form factor functional and an indfinite metric GNS construction and a criterium for the Morchio-Strocchi modified Wightman axioms. In Section 5 we present local, relativistic models for quantum fields with indefinite metric that do not have usual free fields as asymptotics, but dipole fields. In Section 6 we finally show how dipole degrees of freedom occur in the first order perturbation theory of the exponential model. Some technicalities on the asymptotics of certain distributions are given in Appendix A.

\section{Free dipole fields}

Let $d \geq 2$ be the space-time dimension. A free dipole field of mass $m>0$ is a local, relativistic quantum field which fulfills the equations of motion

$$
\left(-\partial_{\nu} \partial^{\nu}-m^{2}\right)^{2} \phi(x)=0
$$

(but $\left(-\partial_{\nu} \partial^{\nu}-m^{2}\right) \phi(x) \neq 0$ in contrast to the usual fee field) and commutation relations

$$
[\phi(x), \phi(y)]_{-}=\frac{\alpha}{i \pi} \Delta_{m}(x-y)+\frac{\beta}{i \pi} \Delta_{m}^{\prime}(x-y)
$$

$\beta \neq 0$,

$$
\Delta_{m}(x)=\frac{i \pi}{(2 \pi)^{d / 2}} \int_{\mathbb{R}^{d}} e^{i k \cdot x}\left(\delta_{m}^{+}(k)-\delta_{m}^{-}(k)\right) d k
$$


and

$$
\Delta_{m}^{\prime}(x)=\frac{i \pi}{(2 \pi)^{d / 2}} \int_{\mathbb{R}^{d}} e^{i k \cdot x}\left(\delta_{m}^{\prime+}(k)-\delta_{m}^{\prime}(k)\right) d k
$$

with

$$
\delta_{m}^{ \pm}(k)=\theta\left( \pm k^{0}\right) \delta\left(k^{2}-m^{2}\right) \quad \text { and } \quad \delta_{m}^{\prime \pm}(k)=\theta\left( \pm k^{0}\right) \delta^{\prime}\left(k^{2}-m^{2}\right) .
$$

Since the two-point function does not admit a Källen-Lehmann representation, dipole fields always are quantum fields with indefinite metric [17, 16].

The equation of motion for dipole fields is a PDE of order 4, hence the dipole field has an additional degree of freedom compared with the usual free field ( note that the the spectral condition rules out 2 degrees of freedom for the dipole field and one degree of freedom for the usual free field. Degree of freedom here means the numbers of functions which have to be specified on a space-like hyperplane to determine the solution).

\section{The one particle wave operator}

In Haag-Ruelle theory [10, 19, 11] asymptotic states are being constructed by definition of the finite time wave operator on the one particle space, which is then extended to the multi-particle states by taking tensor products of the one particle wave operator. By this strategy one circumvents the pitfalls of Haag's theorem and Jost-Schroer theorem (cf. [20]) which say that the interaction picture does not exist. With a similar strategy it is also possible to construct asymptotic states in the case of QFT with indefinite metric [1.

But such asymptotics does not exist in all cases of local, relativistic quantum fields with indefinite metric [5], since scattering amplitudes of some of the models studied in that reference diverge polynomially if one tries to calculate them on the basis of the Haag-Ruelle one particle wave operator. As we will demonstrate below, the asymptotics for such quantum fields is rather given by a free dipole field (at least in the simplest case) than by a usual free field. Thus, taking into account the additional dipole degree of freedom, one can remove this divergence and get a well-defined asymptotics. To carry out this construction, we first have to find the right one particle wave operator for the dipole field.

Such a one particle wave operator $\Omega_{t}^{d \text { in/out }}$ is required to fulfill the following conditions:

(i) $\Omega_{t}^{d \text { in/out }}: \mathcal{S}\left(\mathbb{R}^{d}\right) \rightarrow \mathcal{S}\left(\mathbb{R}^{d}\right)$ acts as a multiplication operator in energy-momentum space (in analogy to the Haag-Ruelle wave operator).

(ii) $\Omega_{t}^{d \text { in/out }}$ leaves free dipole fields invariant, i.e. $\phi \circ \Omega^{d \text { in/out }}(f)=\phi(f) \forall f \in \mathcal{S}\left(\mathbb{R}^{d}\right), t \in$ $\mathbb{R}$.

(iii) For the (interacting) current field $j(x)=\left(-\partial_{\nu} \partial^{\nu}-m^{2}\right) \phi(x)$ the action of $\Omega_{t}^{d \text { in/out }}$ coincides with the action of the usual Haag-Ruelle one-particle wave operator up to a term proportional to $\left(-\partial_{\nu} \partial^{\nu}-m^{2}\right) j(x)$ (i.e. up to a term which vanishes in the free case). 
Let $\varphi \in C_{0}^{\infty}(\mathbb{R}, \mathbb{R})$ with support in $(-\epsilon, \epsilon)$ where $0<\epsilon<m^{2}$ and $\varphi(\kappa)=1$ if $\kappa \epsilon$ $(-\epsilon / 2, \epsilon / 2)$. We define $\chi^{ \pm}(k)=\theta\left( \pm k^{0}\right) \varphi\left(k^{2}-m^{2}\right)$ with $\theta$ the Heaviside step function and we set

$$
\chi_{t}(a, k)= \begin{cases}\chi^{+}(k) e^{-i\left(k^{0}-\omega\right) t}+\chi^{-}(k) e^{-i\left(k^{0}+\omega\right) t} & a=\text { in } \\ 1 & a=\mathrm{loc} \\ \chi^{+}(k) e^{i\left(k^{0}-\omega\right) t}+\chi^{-}(k) e^{i\left(k^{0}+\omega\right) t} & a=\mathrm{out}\end{cases}
$$

with $\omega=\sqrt{|\mathbf{k}|^{2}+m^{2}}$. We then define

$$
\chi_{t}^{d}(\text { in/out }, k)=\left[1 \pm \frac{i t}{2 k^{0}}\left(k^{2}-m^{2}\right)\right] \chi_{t}(\text { in/out }, k)
$$

with the + sign belonging to "in" and the $-\operatorname{sign}$ to "out" and we set $\chi_{t}^{d}($ loc, $k)=1$. We finally define

$$
\Omega_{t}^{d \text { in } / \text { out }}=\mathcal{F} \chi_{t}^{d}(\text { in } / \text { out }, k) \overline{\mathcal{F}}
$$

where $\mathcal{F}(\overline{\mathcal{F}})$ stands for the (inverse) Fourier transform.

$\Omega_{t}^{\text {din/out }}$ fulfills the above items (i) and (iii) by construction. Using Lemma A.3 and Lemma A.4 of the appendix one can verify that also (ii) holds.

Our choice of $\Omega_{t}^{d \text { in/out }}$ is not the only possible one: in particular one can add terms proportional to $p(t) e^{ \pm i\left(k^{0} \pm \omega\right) t}\left(k^{2}-m^{2}\right)^{2}$ (with $p(t)$ a polynomially bounded function) to $\chi_{t}^{d}($ in/out,$k)$ and one obtains another wave operator fulfilling items (i)-(iii) and which gives the same scattering behaviour for the models studied in Section 5. Such "higher order corrections" are needed if one wants to generalize the possible asymptotic behaviour beyond free dipole fields.

\section{Construction of asymptotic states}

Here we give a general scheme for the construction of asymptotic states following [1]:

Let $\underline{\mathcal{S}}$ be the Borchers' algebra over $\mathcal{S}\left(\mathbb{R}^{d}\right)$ and let $\underline{\mathcal{S}}^{\text {ext }}$ be the "extended" Borchers' algebra over the test function space $\mathcal{S}_{1}^{\text {ext }}=\mathcal{S}\left(\mathbb{R}^{d}, \mathbb{C}^{3}\right)$, which is the space of Schwartz functions with values in $\mathbb{C}^{3}$. For $a=$ in/loc/out we define $J^{a}: \mathcal{S}_{1} \rightarrow \mathcal{S}_{1}^{\text {ext }}$ to be the injection of $\mathcal{S}_{1}$ into the first/second/third component of $\mathcal{S}_{1}^{\text {ext }}$, i.e. $J^{\text {in }} f=(f, 0,0), J^{\text {loc }} f=$ $(0, f, 0), J^{\text {out }} f=(0,0, f), f \in \mathcal{S}_{1}$. Then $J^{a}$ uniquely induces a continuous unital $*$-algebra homomorphism $\underline{J}^{a}: \underline{\mathcal{S}} \rightarrow \underline{\mathcal{S}}^{\text {ext }}$ given by $\underline{J}^{a}=\bigoplus_{n=0}^{\infty} J^{a \otimes n}$.

We also define a suitable "projection" $\underline{J}: \underline{\mathcal{S}}^{\text {ext }} \rightarrow \underline{\mathcal{S}}$ as the unique continuous unital *-algebra homomorphism induced by $J: \mathcal{S}_{1}^{\text {ext }} \rightarrow \mathcal{S}_{1}, J\left(f^{\text {in }}, f^{\text {loc }}, f^{\text {out }}\right)=f^{\text {in }}+f^{\text {loc }}+f^{\text {out }}$.

We then define $\Omega_{t}^{d}: \mathcal{S}_{1}^{\text {ext }} \rightarrow \mathcal{S}_{1}^{\text {ext }}$ by

$$
\Omega_{t}^{d}=\left(\begin{array}{ccc}
\Omega_{t}^{d \text { in }} & 0 & 0 \\
0 & 1 & 0 \\
0 & 0 & \Omega_{t}^{d \text { out }}
\end{array}\right)
$$

Next, we introduce the multi parameter $\underline{t}=\left(t_{1}, t_{2}, \ldots\right), t_{n}=\left(t_{n}^{1}, \ldots, t_{n}^{n}\right), t_{n}^{l} \in \mathbb{R}$ and we write $\underline{t} \rightarrow+\infty$ if $t_{n}^{l} \rightarrow+\infty$ in any order, i.e. first one $t_{n}^{l}$ goes to infinity, then the next 
etc. . We say that the limit $\underline{t} \rightarrow+\infty$ of any given object exists, if it exists for $t_{n}^{l} \rightarrow+\infty$ in any order and it does not depend on the order. We now define the finite times wave operator $\underline{\Omega}_{t}^{d}: \underline{\mathcal{S}}^{\text {ext }} \rightarrow \underline{S}$ as

$$
\underline{\Omega}_{t}^{d}=\underline{J} \circ \oplus_{n=0}^{\infty} \Omega_{n, t_{n}}^{d}, \quad \Omega_{0, t_{0}}^{d}=1, \quad \Omega_{n, t_{n}}^{d}=\otimes_{l=1}^{n} \Omega_{t_{n}^{l}}^{d} .
$$

Furthermore, we define the finite times in- and out- wave operators $\underline{\Omega}_{\underline{t}}^{d \text { in/out }}: \underline{\mathcal{S}} \rightarrow \underline{\mathcal{S}}$ as $\underline{\Omega}_{t}^{d} \circ \underline{J}^{\text {in/out}}$. Up to changes of the time parameter which do not matter in the limit $\underline{t} \rightarrow+\infty$, the wave operators $\underline{\Omega}_{t}^{d \text { (in/out) }}$ are $*$-algebra homomorphisms, as can be easily verified from the definitions.

Definition 4.1. (i) Let $\underline{W} \in \underline{\mathcal{S}}^{\prime}$ be a Wightman functional s.t. the functionals $\underline{W} \circ \underline{\Omega}_{t}^{d}$ converge in $\underline{\mathcal{S}}^{\text {ext }}$ as $\underline{t} \rightarrow+\infty$. We then define the dipole form factor functional $\underline{F}^{d} \in \underline{\mathcal{S}}^{\text {ext' }}$ associated to $\underline{W}$ as this limit, i.e.

$$
\underline{F}^{d}=\lim _{\underline{t} \rightarrow+\infty} \underline{W} \circ \underline{\Omega}_{\underline{t}}^{d} .
$$

(ii) The scattering matrix $\underline{S}$ associated to $\underline{W}$ is defined by

$$
\begin{aligned}
\underline{S}(\underline{f}, \underline{g}) & =\underline{F}^{d}\left(\underline{J}^{\text {in }} \underline{f} \otimes \underline{J}^{\text {out }} \underline{g}\right) \\
& =\lim _{\underline{t}, \underline{t}^{\prime} \rightarrow+\infty} \underline{W}\left(\underline{\Omega}_{\underline{t}}^{d \text { in }} \underline{f} \otimes \underline{\Omega}_{\underline{t}^{\prime}}^{\text {out }} \underline{g}\right) \quad \forall \underline{f}, \underline{g} \in \underline{\mathcal{S}} .
\end{aligned}
$$

We then get

Theorem 4.2. We suppose that $\underline{W} \in \underline{\mathcal{S}^{\prime}}$ is the Wightman functional of a local, relativistic QFT with indefininte metric [13, 17]. We assume that the dipole form factor functional $\underline{F}^{d}$ associated to $\underline{W}$ exists and fulfills a Hilbert space structure condition (cf. [13, 17]). Then there exists a quantum field with indefinite metric $\Phi$ (note that the in-loc-and outcomponents of $\Phi$ in general are not local w.r.t. each other) over $\mathcal{S}^{\text {ext }}=\mathcal{S}\left(\mathbb{R}^{d}, \mathbb{C}^{3}\right)$ such that the relativistic quantum fields with indefinite metric $\phi^{\text {in/loc/out }}=\Phi \circ J^{\text {in/loc/out }}$ over $\mathcal{S}\left(\mathbb{R}^{d}\right)$ s.t. $\phi=\phi^{\text {loc }}$ fulfills the LSZ asymptotic condition [15], namely

$$
\lim _{t \rightarrow+\infty} \phi\left(\Omega_{t}^{d \text { in/out }} f\right)=\phi^{\text {in/out }}(f) \quad \forall f \in \mathcal{S}\left(\mathbb{R}^{d}\right)
$$

The idea of the proof is to construct the weak asymptotic limits of the vacuum expectation values first and to do a GNS-like construction of the quantum field $\Phi$ with an indefinite metric state space then. This automatically implies the asymptotic condition (13). For the details see [1].

So far there is no proof for the fact that the in- and out- fields obtained in this way are free dipole fields. Such a general proof requires the generalization of Theorem XI.111 of [18] to the case of dipole fields - in particular we need fast dispersion of functions $h^{t}(x)=\overline{\mathcal{F}}\left(\frac{1}{2 k^{0}} e^{i\left(k^{0}-\omega\right) t}\left(k^{2}-m^{2}\right) \chi^{+}(k) \hat{f}(k)\right)$ in $\mathbf{x}$ as $t \rightarrow \infty$. $\left\|h^{t}\left(x^{0}, .\right)\right\|_{\mathbf{x}} \sim t^{-5 / 2}$ for a suitable Schwartz norm $\|\cdot\|_{\mathbf{x}}$ on $\mathcal{S}\left(\mathbb{R}^{d-1}\right)$ is sufficient. This can be obtained from the usual dispersion $\sim t^{-3 / 2}($ for $d=4)$ for functions $h^{t}(x)=\overline{\mathcal{F}}\left(\frac{1}{2 k^{0}} e^{i\left(k^{0}-\omega\right) t}\left(k^{0}+\omega\right) \chi^{+}(k) \hat{f}(k)\right)$ by differentiation w.r.t. $t$. In the cases we study in the following section one can prove that the asymptotic fields are free dipole fields by explicit calculations. 


\section{Models of interacting dipole fields}

Let $\psi(t), t \in \mathbb{R}$, be an infinitely differentiable Lévy characteristic [9] and let $c=$ $-\left.\left(d^{2} \psi(t) / d t^{2}\right)\right|_{t=0}$. Let $\eta(x)$ be a ultralocal "noise" field over the Euclidean space-time $\mathbb{R}^{d}$ with functional Fourier transform (cf. e.g. [14] for the definition of random fields via functional Fourier transforms)

$$
C_{F}(f)=\exp \left\{\int_{\mathbb{R}^{d}} \psi(f)-\frac{c}{m^{2}}\langle\nabla f, \nabla f\rangle-\frac{c}{m^{4}}\left\langle\nabla f,\left(-\Delta+m^{2}\right) \nabla f\right\rangle d x\right\}
$$

We consider the stochastic partial differential equation (SPDE)

$$
\left(-\Delta+m^{2}\right)^{2} \varphi=\eta
$$

which clearly is a Euclidean dipole type equation driven by the noise field $\eta$. Using standard methods (cf. [2, 7]) one can solve this equation and calculate the moment functions of $X$ :

$$
\left\langle\varphi\left(x_{1}\right) \cdots \varphi\left(x_{n}\right)\right\rangle=\sum_{I \in \mathcal{P}^{(n)}} \prod_{\left\{j_{1}, \ldots, j_{l}\right\} \in I} S_{n}^{T}\left(x_{j_{1}}, \ldots, x_{j_{l}}\right)
$$

where $\mathcal{P}^{(n)}$ is the collection of partitions of $\{1, \ldots, n\}$ into disjoint subsets and the truncated Schwinger functions $S_{n}^{T}$ are given by

$$
S_{2}^{T}(x, y)=\frac{c}{m^{4}}\left(-\Delta+m^{2}\right)^{-2}(x-y)
$$

and for $n \geq 3$

$$
S_{n}^{T}\left(x_{1}, \ldots, x_{n}\right)=c_{n} \int_{\mathbb{R}^{d}} \prod_{l=1}^{n}\left(-\Delta+m^{2}\right)^{-2}\left(x-x_{l}\right) d x
$$

where $c_{n}=\left.i^{-n}\left(d^{n} \psi(t) / d t^{n}\right)\right|_{t=0}$. The analytic continuation of these truncated Schwinger functions from imaginary Euclidean time to real relativistic time has been performed in [5]. We get for the Fourier transforms of the truncated Wightman functions

$$
\hat{W}_{2}^{T}\left(k_{1}, k_{2}\right)=-\frac{c}{m^{4}} \delta_{m}^{\prime}-\left(k_{1}\right) \delta\left(k_{1}+k_{2}\right)
$$

and

$$
\hat{W}_{n}^{T}\left(k_{1}, \ldots, k_{n}\right)=(-1)^{n} \tilde{c}_{n}\left\{\sum_{j=1}^{n} \prod_{l=1}^{j-1} \delta_{m}^{\prime}-\left(k_{l}\right) \frac{1}{\left(k^{2}-m^{2}\right)^{2}} \prod_{l=j+1}^{n} \delta_{m}^{\prime+}\left(k_{l}\right)\right\} \delta\left(\sum_{l=1}^{n} k_{l}\right)
$$

with $\tilde{c}_{n}=(2 \pi)^{\frac{d(n-2)-2}{2}} c_{n}$.

$1 /\left(k^{2}-m^{2}\right)^{2}$ has to be understood in the sense of Cauchy's principal value.

It has been proven in [5] that the truncated Wightman functions $W_{n}^{T}=\overline{\mathcal{F}}\left(\hat{W}_{n}^{T}\right)$ are the truncated vacuum expectation values of a local, relativistic QFT with indefinite metric. 
In the same reference it has been proven that the usual form factor functional of the associated Wightman functional does not exist since Haag-Ruelle like scattering amplitudes diverge poynomially in time. Here we show that the asymptotic behaviour of the given QFT with indefinite metric is rather given by free dipole fields.

Using Lemma A.4 and A.6 one gets for $n \geq 3$ that

$$
\lim _{t_{n}^{1}, \ldots, t_{n}^{n} \rightarrow+\infty} \prod_{l=1}^{n} \chi_{t_{n}^{l}}^{d}\left(a_{l}, k_{l}\right) \hat{W}_{n}^{T}\left(k_{1}, \ldots, k_{n}\right)=\hat{F}_{n}^{d, T\left(a_{1}, \ldots, a_{n}\right)}\left(k_{1}, \ldots, k_{n}\right),
$$

where

$$
\hat{F}_{2}^{d, T\left(a_{1}, a_{2}\right)}\left(k_{1}, k_{2}\right)=\hat{W}_{2}^{T}\left(k_{1}, k_{2}\right)
$$

and for $n \geq 3$

$$
\begin{aligned}
& \hat{F}_{n}^{d, T\left(a_{1}, \ldots, a_{n}\right)}\left(k_{1}, \ldots, k_{n}\right) \\
= & \left\{\sum_{j=1}^{n} \prod_{l=1}^{j-1} \delta_{m}^{\prime-}\left(k_{l}\right) \hat{\Delta}_{m}^{\prime}\left(a_{j}, k_{j}\right) \prod_{l=j+1}^{n} \delta_{m}^{\prime}\left(k_{l}\right)\right\} \delta\left(\sum_{l=1}^{n} k_{l}\right),
\end{aligned}
$$

$\left(a_{l}=\right.$ in/loc/out $)$, and

$$
\hat{\Delta}_{m}^{\prime}(a, k)= \begin{cases}i \pi\left(\delta_{m}^{\prime}(k)-\delta_{m}^{\prime}-(k)\right) & \text { for } a=\text { in } \\ 1 /\left(k^{2}-m^{2}\right)^{2} & \text { for } a=\text { loc } \\ -i \pi\left(\delta_{m}^{\prime}(k)-\delta_{m}^{\prime}-(k)\right) & \text { for } a=\text { out }\end{cases}
$$

We summarize our discussion with the following theorem:

Theorem 5.1. Let $\underline{W}$ be the Wightman functional associated to the Fourier transformed, truncated Wightman functions defined above. Then

(i) $\underline{W}$ fulfills the modified Wightman axioms of Morchio and Strocchi [17].

(ii) The usual form factor functional for $\underline{W}$ does not exist, but the dipole form factor functional exists and fulfills a Hilbert space structure condition.

(iii) Thus, there exist a local, relativistic quantum field $\phi(x)$ with indefinite metric such that the local quantum field converges to asymptotic fields $\lim _{t \rightarrow \infty} \phi \circ \Omega_{t}^{d \text { in/out }}(f)=\phi^{\text {in/out }}(f) \forall f \in$ $\mathcal{S}\left(\mathbb{R}^{d}\right)$.

(iv) The asymptotic fields $\phi^{\text {in/out }}(x)$ fulfill the equation of motion and commutation relations of the free dipole fields.

(v) The S-matrix associated to the quantum field $\phi(x)$ is given by

$$
\begin{aligned}
& \left\langle\phi^{\text {in }}\left(k_{r}\right) \cdots \phi^{\text {in }}\left(k_{1}\right) \Psi_{0}, \phi^{\text {out }}\left(k_{r+1}\right) \cdots \phi^{\text {out }}\left(k_{n}\right) \Psi_{0}\right\rangle^{T} \\
= & 2 \pi i \tilde{c}_{n} \prod_{l=1}^{n} \delta_{m}^{\prime}\left(k_{l}\right) \delta\left(\sum_{l=1}^{r} k_{l}-\sum_{l=r+1}^{n} k_{l}\right),
\end{aligned}
$$

where $k_{1}^{0}, \ldots, k_{n}^{0}>0$ (i.e. all $\phi^{\text {in/out }}\left(k_{l}\right)$ are creation operators), $\Psi_{0}$ is the vacuum state and $n \geq 3$. 
Proof. (i) and (ii): This has been established in the above discussion, that the form factor functional fulfills the Hilbert space structure condition follows from a slight generalization of Lemma 4.9 of [1].

(iii) is a consequence of Theorem 4.2 ,

(iv) and (v) can be verified by calculations using the explicit formulae for the truncated form factor functional (for similar calculations in a related case however with usual free asymptotic fields cf. [1]) .

We note that there are strong indications that for fields with a usual (non dipole) form factor functional $\underline{F}[1]$ also the dipole form factor functional $\underline{F}^{d}$ exists and that $\underline{F}=\underline{F}^{d}$ in this case. Using the Lemmas A.3 and A.5 below, this e.g. can be verified for the class of models studied in [1].

\section{Dipoles in perturbation theory}

In this section we biefly show, how dipole degrees of freedom occur in the low order perturbation theory for the exponential [12] or trigonometric [6] model in $d=2$. Although these models after analytic continuation fulfill the Wightman axioms and thus have a well-defined scattering theory without any dipoles showing up, already in the first order contribution to the Wightman function we do get a dipole term which, however, does not contribute to the non-trivial part of the scattering amplitude if dipole asymtotics is taken into account. If not, the scattering behavior of the first order contribution is ill defined.

Let $\langle A\rangle=\int_{\mathcal{S}^{\prime}\left(\mathbb{R}^{2}\right)} A(\varphi) d \mu_{0}(\varphi)$ be the expected value of $A$ with respect to the free field measure $\mu_{0}$ on $\mathcal{S}^{\prime}\left(\mathbb{R}^{2}\right)$, i.e. the measure with characteristic functional

$$
\mathcal{C}(f)=\exp \left\{-\frac{1}{2}\left(f,\left(-\Delta+m^{2}\right)^{-1} f\right)\right\}, f \in \mathcal{S}\left(\mathbb{R}^{2}\right) .
$$

It is well-known that for $\rho$ a probability measure with support in $(-\sqrt{4 \pi}, \sqrt{4 \pi})$ and $v(t)=\int_{\mathbb{R}} e^{\alpha t} d \rho(\alpha)$, the exponential interaction $\int_{\Lambda}: v(\varphi): d x$ for $\Lambda \subseteq \mathbb{R}^{2}$ compact is a positive function and square integrable with respect to $\mu_{0}$. We define the Schwinger functions of the interacting meausre with infra red cut-off $\Lambda$ as

$$
\left\langle\varphi\left(x_{1}\right) \cdots \varphi\left(x_{n}\right)\right\rangle_{\Lambda, \lambda}=\frac{\left\langle\varphi\left(x_{1}\right) \cdots \varphi\left(x_{n}\right) e^{-\lambda \int_{\Lambda}: v(\varphi): d x}\right\rangle}{\left\langle e^{-\lambda \int_{\Lambda}: v(\varphi): d x}\right\rangle}, \quad \lambda \geq 0 .
$$

Since the potential term is square intrgrable, the Schwinger functions for $\lambda \geq 0$ are twice differentiable (at $\lambda=0$ differentiable from the right) which gives us the first order expansion together with a control of the error due to Taylors lemma. Using also $(a-$ $\lambda b) /(1-\lambda c)=a-\lambda b+\lambda a c+o\left(\lambda^{2}\right)$ and $\langle: \exp \alpha \varphi(x):\rangle=1$, we obtain

$$
\begin{aligned}
\left\langle\varphi\left(x_{1}\right) \cdots \varphi\left(x_{n}\right)\right\rangle_{\Lambda, \lambda} & =\left\langle\varphi\left(x_{1}\right) \cdots \varphi\left(x_{n}\right)\right\rangle \\
& -\frac{\lambda}{2} \int_{\mathbb{R}} \int_{\Lambda}\left\langle\varphi\left(x_{1}\right) \cdots \varphi\left(x_{n}\right): \exp \alpha \varphi(x):\right\rangle d x d \rho(\alpha) \\
& +\frac{\lambda}{2}\left\langle\varphi\left(x_{1}\right) \cdots \varphi\left(x_{n}\right)\right\rangle|\Lambda|+o\left(\lambda^{2}\right)
\end{aligned}
$$


where $|\Lambda|$ is the volume of $\Lambda$. By a standard calculation

$$
\left\langle: \exp \alpha \varphi(x): e^{i \varphi(f)}\right\rangle=\exp \left[i \alpha\left(-\Delta+m^{2}\right)^{-1} f(x)-\frac{1}{2}\left(f,\left(-\Delta+m^{2}\right)^{-1} f\right)\right] .
$$

Performing functional derivatives with respect to $f$ at $f=0$ and multiplying by $(-i)^{n}$, one obtains

$$
\begin{aligned}
\left\langle\varphi\left(x_{1}\right) \cdots \varphi\left(x_{n}\right): \exp \alpha \varphi(x):\right\rangle= & \sum_{S \subseteq\{1, \ldots, n\}} \alpha^{|S|} \prod_{j \in S}\left(-\Delta+m^{2}\right)^{-1}\left(x_{j}-x\right) \\
\times & \sum_{I \in \mathcal{P}_{2}(\{1, \ldots, n\} \backslash S)} \prod_{\{j, l\} \in I}\left(-\Delta+m^{2}\right)^{-1}\left(x_{j}-x_{l}\right)
\end{aligned}
$$

Here $\mathcal{P}_{2}(A)$ is the collection of all pair partitions of the set $A$. $|S|$ gives the cardinaliy of $S$.

Let us first consider the contribution to (28) that stems from those terms in (30) with $S=\emptyset$. Appearently those terms give a contriution $-\frac{\lambda}{2}|\Lambda|\left\langle\varphi\left(x_{1}\right) \cdots \varphi\left(x_{n}\right)\right\rangle$ that cancels the term in the last line of that equation. Removing the infra red cut-off via $\Lambda \nearrow \mathbb{R}^{2}$, one obtains

$$
\begin{aligned}
& \left\langle\varphi\left(x_{1}\right) \cdots \varphi\left(x_{n}\right)\right\rangle_{\lambda}=\sum_{\emptyset \neq S \subseteq\{1, \ldots, n\}} \int_{\mathbb{R}} \alpha^{|S|} d \rho(\alpha) \int_{\mathbb{R}^{2}} \prod_{j \in S}\left(-\Delta+m^{2}\right)^{-1}\left(x_{j}-x\right) d x \\
& \times \sum_{I \in \mathcal{P}_{2}(\{1, \ldots, n\} \backslash S)} \prod_{\{j, l\} \in I}\left(-\Delta+m^{2}\right)^{-1}\left(x_{j}-x_{l}\right)+o\left(\lambda^{2}\right)
\end{aligned}
$$

For $S=\left\{s_{1}, \ldots, s_{q}\right\}$ and $I$ fixed, we can now do the analytic continuations factor by factor. The Fourier transformed analytic continuation of the pairing terms just gives the usual to point function $\delta_{m}^{-}\left(k_{j}\right) \delta\left(k_{j}+k_{l}\right)(j<l)$. The first factor is a constant for $q=1$. For $q=2, S=\left\{s_{1}, s_{2}\right\}$ we get (17) up to a constant and hence the Fourier transformed analytic continuation up to a constant factor is given by (19), which is a dipole two point function. If $q \geq 3$, by [2] one obtains as the Fourier transformed analytic continuation

$$
\tilde{c}_{q}\left\{\sum_{j=1}^{q} \prod_{l=1}^{j-1} \delta_{m}^{-}\left(k_{s_{l}}\right) \frac{1}{\left(k_{s_{j}}^{2}-m^{2}\right)} \prod_{l=j+1}^{q} \delta_{m}^{+}\left(k_{s_{l}}\right)\right\} \delta\left(\sum_{l=1}^{q} k_{s_{l}}\right) .
$$

Here $c_{n}$ in the definition of $\tilde{c}_{n}$ is replaced by the $n$-th moment of $\rho$. Appearently, the dipole degrees of freedom here do not contribute to scattering, if the proper dipole asymptotics is taken into account. Following the argument of section 5, one can show that the contribution of (32) to the dipole scattering amplitude is (see also [1])

$$
2 \pi i \tilde{c}_{q} \prod_{l=1}^{q} \delta_{m}^{+}\left(k_{s_{l}}\right) \delta\left(\sum_{l=1}^{r} k_{s_{l}}-\sum_{l=r+1}^{q} k_{s_{l}}\right)
$$

i.e. the non trivial scattering concerns the classicle particle degrees of freedom, only. 
In fact, (33) is just what one would expect from the expansion : $\exp \alpha \varphi(x):=\sum_{l=0}^{\infty} \frac{\alpha^{l}}{l !}$ : $\varphi^{l}(x)$ : with the first order term cancelled by a symmetric choice of $\rho\left(\rho=\frac{1}{2}\left(\delta_{-1}+\delta_{1}\right)\right.$ for massive sinh-Gordon) and the second order term absorbed into a re-definition of the the mass, which however in the rigorous formulation of the exponential model is not needed. In fact, in that expansion any $: \varphi^{l}$ : interaction term to first order corresponds to a pure s-wave scattering (in terms of Feynman graphs: a star graph with $l$ legs) which is just (33). We have thus seen that the dipole formulation of scattering allows us to get the correct 1st order scattering amplitude without any unnecessary re-definition of the mass.

Here the calculations have been carried out for the exponential model. For the trigonometric model the argument is analogous with the only difference that $\alpha$ is replaced by $i \alpha$.

\section{A Some distributional identities}

Let $m>0, d \geq 2, \partial_{0}=\left(\partial / \partial k^{0}\right), \omega=\sqrt{|\mathbf{k}|^{2}+m^{2}}$.

Lemma A.1. $\delta_{m}^{\prime \pm}(k)=\frac{1}{2 k^{0}} \partial_{0} \delta_{m}^{ \pm}(k)$.

Proof. By change of variables.

Lemma A.2. $\left(k^{2}-m^{2}\right) \delta_{m}^{\prime \pm}(k)=-\delta_{m}^{ \pm}(k)$.

Proof. By Lemma A.1

$$
\begin{aligned}
\int_{\mathbb{R}^{d}}\left(k^{2}-m^{2}\right) \delta_{m}^{\prime \pm}(k) \hat{f}(k) d k & =-\int_{\mathbb{R}^{d}} \delta_{m}^{ \pm}(k) \partial_{0}\left[\frac{\left(k^{2}-m^{2}\right)}{2 k^{0}} \hat{f}(k)\right] d k \\
& =-\int_{\mathbb{R}^{d}} \delta_{m}^{ \pm}(k)\left[\frac{\partial_{0}\left(k^{2}-m^{2}\right)}{2 k^{0}}\right] \hat{f}(k) d k
\end{aligned}
$$

Since $\left[\frac{\partial_{0}\left(k^{2}-m^{2}\right)}{2 k^{0}}\right]_{k^{0}= \pm \omega}=1$, the claim follows.

Lemma A.3. $\chi_{t}^{d}(a, k) \delta_{m}^{ \pm}(k)=\delta_{m}^{ \pm}(k)$.

Proof. Let $a=$ out ( $a=\operatorname{loc}$ is trivial and $a=$ in can be treated analogously), then

$$
\begin{aligned}
\int_{\mathbb{R}^{d}} \delta_{m}^{ \pm}(k) \chi_{t}^{d}(\text { out }, k) \hat{f}(k) d k & =\int_{\mathbb{R}^{d}} \delta_{m}^{ \pm}(k) e^{i\left(k^{0} \mp \omega\right) t} \\
& \times\left[1-\frac{i t}{2 k^{0}}\left(k^{2}-m^{2}\right)\right] \chi^{ \pm}(k) \hat{f}(k) d k \\
& =\int_{\mathbb{R}^{d}} \delta_{m}^{ \pm}(k) \hat{f}(k) d k
\end{aligned}
$$

Lemma A.4. $\chi_{t}^{d}(a, k) \delta_{m}^{\prime \pm}(k)=\delta_{m}^{\prime \pm}(k)$. 
Proof. As in Lemma A.3, it suffices to consider $a=$ out:

$$
\begin{aligned}
& \int_{\mathbb{R}^{d}} \delta_{m}^{\prime \pm}(k) \chi_{t}^{d}(\text { out }, k) \hat{f}(k) d k \\
= & \int_{\mathbb{R}^{d}} \delta_{m}^{\prime \pm}(k) e^{i\left(k^{0} \mp \omega\right) t}\left[1-\frac{i t}{2 k^{0}}\left(k^{2}-m^{2}\right)\right] \chi^{ \pm}(k) \hat{f}(k) d k \\
= & -\int_{\mathbb{R}^{d}} \delta_{m}^{ \pm}(k) \frac{i t}{2 k^{0}}\left[1-\frac{i t}{2 k^{0}}\left(k^{2}-m^{2}\right)\right] \chi^{ \pm}(k) \hat{f}(k) d k \\
+ & \int_{\mathbb{R}^{d}} \delta_{m}^{\prime \pm}(k)\left[1-\frac{i t}{2 k^{0}}\left(k^{2}-m^{2}\right)\right] \chi^{ \pm}(k) \hat{f}(k) d k \\
= & \int_{\mathbb{R}^{d}} \delta_{m}^{\prime \pm}(k) \hat{f}(k) d k
\end{aligned}
$$

Here we used Lemma A.2 in the last step.

Lemma A.5. $\lim _{t \rightarrow+\infty} \frac{\chi_{t}^{d}(a, k)}{\left(k^{2}-m^{2}\right)}=\hat{\Delta}_{m}(a, k)$ (for the definition of $\hat{\Delta}_{m}(a, k) c f$. [1]).

Proof. Let $a=$ in/out ( $a=$ loc is trivial). Then

$$
\begin{aligned}
\lim _{t \rightarrow+\infty} \int_{\mathbb{R}^{d}} \frac{\chi_{t}^{d}(a, k)}{\left(k^{2}-m^{2}\right)} \hat{f}(k) d k & =\lim _{t \rightarrow \infty}\left[\int_{\mathbb{R}^{d}} \frac{\chi_{t}(a, k)}{\left(k^{2}-m^{2}\right)} \hat{f}(k) d k\right. \\
& \left.-i t \int_{\mathbb{R}^{d}} \frac{\chi_{t}(a, k)}{2 k^{0}} \hat{f}(k) d k\right]
\end{aligned}
$$

The second integral on the right hand side vanishes faster than any inverse polynomial in $t$ as $t \rightarrow+\infty$, thus the statement follows from Lemma 4.8 of [1].

Lemma A.6. $\lim _{t \rightarrow+\infty} \frac{\chi_{t}^{d}(a, k)}{\left(k^{2}-m^{2}\right)^{2}}=\hat{\Delta}_{m}^{\prime}(a, k)$.

Proof. For $a=$ out we calculate $(a=$ in can be treated analogously and $a=$ loc is trivial)

$$
\begin{aligned}
& \int_{\mathbb{R}^{d}} \frac{\chi_{t}^{d}(\text { out }, k)}{\left(k^{2}-m^{2}\right)^{2}} \hat{f}(k) d k \\
= & \int_{\mathbb{R}^{d}} \frac{1}{k^{2}-m^{2}} \partial_{0}\left[\frac{1-\frac{i t}{2 k^{0}}\left(k^{2}-m^{2}\right)}{2 k^{0}} \chi_{t}(\text { out }, k) \hat{f}(k)\right] d k \\
= & \int_{\mathbb{R}^{d}} \frac{\chi_{t}(\text { out }, k)}{\left(k^{2}-m^{2}\right)} \partial_{0}\left[\frac{1-\frac{i t}{2 k^{0}}\left(k^{2}-m^{2}\right)}{2 k^{0}} \hat{f}(k)\right] d k \\
+ & i t \int_{\mathbb{R}^{d}} \frac{\chi_{t}(\text { out }, k)}{\left(k^{2}-m^{2}\right)} \frac{1-\frac{i t}{2 k^{0}}\left(k^{2}-m^{2}\right)}{2 k^{0}} \hat{f}(k) d k \\
+ & \int_{\mathbb{R}^{d}} \frac{e^{i\left(k^{0}-\omega\right) t} \partial_{0} \chi^{+}(k)+e^{i\left(k^{0}+\omega\right) t} \partial_{0} \chi^{-}(k)}{\left(k^{2}-m^{2}\right)} \\
\times & \frac{1-\frac{i t}{2 k^{0}}\left(k^{2}-m^{2}\right)}{2 k^{0}} \hat{f}(k) d k
\end{aligned}
$$


The third integral on the right hand side vanishes faster than any inverse polynomial as $t \rightarrow \infty$. For the first two integrals we get

$$
\begin{aligned}
& \int_{\mathbb{R}^{d}} \frac{\chi_{t}(\text { out }, k)}{\left(k^{2}-m^{2}\right)} \partial_{0}\left[\frac{1}{2 k^{0}} \hat{f}(k)\right] d k \\
+ & i t \int_{\mathbb{R}^{d}} \frac{\chi_{t}(\text { out }, k)}{\left(k^{2}-m^{2}\right)} \frac{1}{2 k^{0}}\left[1-\frac{\partial_{0}\left(k^{2}-m^{2}\right)}{2 k^{0}}\right] \hat{f}(k) d k \\
- & i t \int_{\mathbb{R}^{d}} \chi_{t}(\text { out }, k) \partial_{0}\left[\frac{1}{\left(2 k^{0}\right)^{2}} \hat{f}(k)\right] d k \\
+ & t^{2} \int_{\mathbb{R}^{d}} \chi_{t}(\text { out }, k) \frac{1}{\left(2 k^{0}\right)^{2}} \hat{f}(k) d k .
\end{aligned}
$$

The last two integrals on the right hand side vanish faster than any inverse polynomial as $t \rightarrow \infty$. This holds also true for the second integral, since by Lemma 4.8 of [1] the limit of the integral is

$$
\int_{\mathbb{R}^{d}} \frac{\hat{\Delta}_{m}(\text { out }, k)}{2 k^{0}}\left[1-\frac{\partial_{0}\left(k^{2}-m^{2}\right)}{2 k^{0}}\right] \hat{f}(k) d k=0
$$

and furthermore

$$
\frac{d}{d t} \int_{\mathbb{R}^{d}} \frac{\chi_{t}(\text { out }, k)}{\left(k^{2}-m^{2}\right)} \frac{1}{2 k^{0}}\left[1-\frac{\partial_{0}\left(k^{2}-m^{2}\right)}{2 k^{0}}\right] \hat{f}(k) d k \rightarrow 0
$$

faster than any inverse polynomial as $t \rightarrow+\infty$. Now the claim follows from Lemma 4.8 of [1] and integration by parts.

Acknowledgements. I gratefully acknowledge valuable discussions with S. Albeverio, F. Strocchi, J.-L. Wu and G. Lechner.

\section{References}

[1] S. Albeverio, H. Gottschalk, Scattering theory for quantum fields with indefinite metric, Commun. Math. Phys. 216 (2001), 491-513.

[2] S. Albeverio, H. Gottschalk, J.-L. Wu, Convoluted generalized white noise, Schwinger functions and their continuation to Wightman functions, Rev. Math Phys., Vol 8, No. 6 (1996), 763-817.

[3] S. Albeverio, H. Gottschalk, J.-L. Wu, Models of local relativistic quantum fields with indefinite metric (in all dimensions), Commun. Math. Phys. 184 (1997), 509-531.

[4] S. Albeverio, H. Gottschalk, J.-L. Wu, Nontrivial scattering amplitudes for some local relativistic quantum field models with indefinite metric, Phys. Lett. B 405 (1997), 243248.

[5] S. Albeverio, H. Gottschalk, J.-L. Wu, Scattering behaviour of quantum vector fields obtained from Euclidean covariant SPDEs, Rep. on Math. Phys. 44 No. 1/2 (1999), 21-28. 
[6] S. Albeverio, R. Høegh-Krohn, Uniqueness of the physical vacuum and the Wightman functions in the infinite volume limit for some non polynomial interactions, Commun. Math. Phys. 30 (1973), 171-200.

[7] S. Albeverio, K. Iwata, T. Kolsrud, Random fields as solutions of the inhomogenous quarternionic Cauchy-Riemann equation. I. Invariance and analytic continuation, Commun. Math. Phys. 132 (1990), 555-580.

[8] C. Becker, R. Gielerak, P. Ługiewicz, Covariant SPDEs and quantum field structures, J. Phys. A 31 (1998), 231-258.

[9] C. Berg, G. Forst, Potential Theory on Locally Compact Abelian Groups. Berlin/Heidelberg/New York: Springer-Verlag 1975.

[10] R. Haag, Quantum field theories with composite particles and aymptotic condition, Phys. Rev. 112 (1958), 669-673.

[11] K. Hepp, On the connection between the LSZ and Wightman quantum field theory, Commun. Math. Phys. 1 (1965), 95-111.

[12] R. Høegh-Krohn, A general class of quantum fields without cut-offs in two space-time dimensions, Comm. Math. Phys. 21 (1971), 244-255.

[13] G. Hoffmann, On GNS representations on inner product spaces: I. The structure of the representation space, Commun. Math. Phys., 191 (1998), 299-323.

[14] K. Itô, Foundations of S stochastic differential equations in infinite dimensional spaces, CBMS-NSF Regional Conf. Series in Applied Math. 47, Philadelphia: SIAM 1984.

[15] H. Lehmann, K. Symanzik, W. Zimmermann, Zur Formulierung quantisierter Feldtheorien, Il Nuovo Cimento 1 (1954), 205-225.

[16] U. Moschella, F. Strocchi, The dipole field model, Lett. Math. Phys. 19(1990), 143-149.

[17] G. Morchio, F. Strocchi, Infrared singularities, vacuum structure and pure phases in local quantum field theory, Ann. Inst. H. Poincaré, Vol. 33 (1980), 251-282.

[18] M. Reed, B. Simon, Methods of modern mathematical physics Vol.II+ III: Academic Press, San Diego, 1979.

[19] D. Ruelle, On the asymptotic condition in quantum field theory, Helv. Phys. Acta 35 (1962), 147-163.

[20] R.F. Streater, A.S. Wightman, PCT, spin and statistics, and all that, Benjamin, New York, Amsterdam, 1964.

[21] O. Steinmann, Perturbative quantum electrodynamics and axiomatic field theory, Springer Verlag, Berlin/Heidelberg/New York, 2000.

[22] F. Strocchi, Selected topics on the general properties of quantum field theory, Lecture Notes in Physics 51, Singapore-New York-London-Hong Kong: World Scientific, 1993. 